\title{
Imaging Chladni figure of plasmonic charge density wave in real
}

\section{space}

Zhaoshuai Gao ${ }^{1}$, Lixin Yin ${ }^{1}$, Weina Fang 2 , Qingying Kong ${ }^{1}$, Chunhai Fan²,

Bin Kang ${ }^{1 \star}$, Jing-Juan $\mathrm{Xu}^{1}$, Hong-Yuan Chen ${ }^{1}$

${ }^{1}$ State Key Laboratory of Analytical Chemistry for Life Science and Collaborative Innovation Center of Chemistry for Life Sciences, School of Chemistry and Chemical Engineering, Nanjing University, 163 Xianlin Road, Nanjing 210023, China.

${ }^{2}$ School of Chemistry and Chemical Engineering, and Institute of Molecular Medicine, Renji Hospital, School of Medicine, Shanghai Jiao Tong University, Shanghai 200240, China

${ }^{\star}$ Correspondence should be addressed to binkang@nju.edu.cn (B. K.)

\section{Supplementary contents:}
I. Theory and modeling
II. Supplementary figures
III. Supplementary movies 


\section{Theory and modeling}

\section{$\underline{\text { Reflection index and electron density }}$}

Based on Maxwell equations and Drude's free electron model ${ }^{1}$, when a plane electromagnetic wave $\boldsymbol{E}(\boldsymbol{r}, t)$ sheds on a metal, we can deduce the vector wave equation of free electron gas in the metal as:

$$
\nabla^{2} \boldsymbol{E}(\boldsymbol{r}, t)-\mu_{0} \sigma \frac{\partial \boldsymbol{E}(\boldsymbol{r}, t)}{\partial t}-\varepsilon_{0} \mu_{0} \frac{\partial^{2} \boldsymbol{E}(\boldsymbol{r}, t)}{\partial t^{2}}=0 .
$$

In this equation, the spatial vector is $\boldsymbol{r}=(x, y, z), \mu_{0} 、 \varepsilon_{0}$ are the magnetic permeability and permittivity in vacuum. $\sigma$ is the conductivity under alternative electronic field and $t$ is time. A plane wave equation with the wave vector $\boldsymbol{k}$, angular frequency $\omega$ and the initial phase $\varphi$ can be written as:

$$
\boldsymbol{E}(\boldsymbol{r}, t)=\boldsymbol{E}_{\mathbf{0}} e^{i(\boldsymbol{k} \cdot \boldsymbol{r}-\omega t+\varphi)} .
$$

Additionally, conductivity under alternative electronic field can be written as $\sigma=$ $\frac{\sigma_{0}}{1-i \omega \tau}$, in which the direct current conductivity is $\sigma_{0}=\frac{\rho e^{2} \tau}{m} . \rho, \tau, \mathrm{e}, m, \varphi$ are the density of free electrons, relax time of free electrons, electrons charge, effective mass of free electrons and the phase of the incident light, respectively.

Substituting equation (2) into equation (1), and considering plasmon oscillating frequency $\omega_{p}^{2}=\frac{\rho e^{2}}{\varepsilon_{0} m}$, we can deduce the relative permittivity of metal as:

$$
\varepsilon_{m}=1-\frac{\omega_{p}^{2}}{\omega^{2}+\tau^{-2}}+i \frac{\omega_{p}^{2} \tau}{\omega\left(1+\omega^{2} \tau^{2}\right)}
$$

which could be simplified written as $\varepsilon_{m}=\varepsilon_{m}^{\prime}+i \varepsilon_{m}^{\prime \prime}$. On the condition visible light incident, the relative permittivity of metal can be written as:

$$
\varepsilon_{m}=1-\frac{\omega_{p}^{2}}{\omega^{2}}
$$

And the refractive index of metal is $\mathrm{n}=\sqrt{\varepsilon_{m}}$, the complex refractive index can be 
written as:

$$
n=n^{\prime}+n^{\prime \prime} i .
$$

According to Fresnel's law, the reflection index, $R$ on the metal surface with the surrounding refractive index $n_{1}$ can be written as:

$$
R=\frac{\left(n^{\prime}-n_{1}\right)^{2}+n^{\prime \prime 2}}{\left(n^{\prime}+n_{1}\right)^{2}+n^{\prime \prime 2}}
$$

The scattering light power $P_{s c a}$ is the product of reflection index and incident light power $P_{\text {in }}$, then we have

$$
P_{\text {sca }}=P_{\text {in }} \cdot R
$$

From these analyses and theoretical derivations, we can know that when light shed on the gold triangle, free electrons oscillate along with the electronic field component of light, which generate a 2D standing plasmonic charge density wave (p-CDW) and result in redistribution of the free electrons. As the refraction index of metal is the square root of relative permittivity $n=\sqrt{\varepsilon_{m}}$ and the relative permittivity is rooted in charge density $\varepsilon_{m}=1-\frac{\rho e^{2}}{\omega^{2} \varepsilon_{0} m}$, the origin of reflection index of metal $R$ is charge density.

\section{Local electronic field induced by the incident light on metal plasmon surface}

To describe the focused light spot $\boldsymbol{E}_{\mathbf{0}}\left(\boldsymbol{R}_{\mathbf{0}}, \boldsymbol{r}, \omega\right)$ on the interface between two materials having different refractive indices at the point $r=(\mathrm{x}, \mathrm{y}, \mathrm{z})$, based on the focused light on high numerical aperture objective model, we can write the excitation light as follows ${ }^{2}$

$$
\begin{gathered}
\boldsymbol{E}_{\mathbf{0}}\left(\boldsymbol{R}_{\mathbf{0}}, \boldsymbol{r}, \omega\right)= \\
\int_{-\sqrt{\varepsilon_{1} k}}^{\sqrt{\varepsilon_{1} k}} d \alpha \int_{-\sqrt{\varepsilon_{1} k^{2}-\alpha^{2}}}^{\sqrt{\varepsilon_{1} k^{2}-\alpha^{2}}} d \beta \times \zeta \exp \left[-\frac{w_{0}^{2}\left(\alpha^{2}+\beta^{2}\right)}{4}\right] \exp \left[i \alpha\left(x-x_{0}\right)+i \beta\left(y-y_{0}\right)+i k_{z}\left(z-z_{0}\right)\right]
\end{gathered}
$$


In which $\boldsymbol{R}_{\mathbf{0}}=\left(x_{0}, y_{0}, z_{0}\right)$ is the coordinate of the incident light, $\varepsilon_{1}$ is the permittivity of the imaging surrounding, $w_{0}$ is the waist of the incident light. The wave vector is $k^{2}$ $=\alpha^{2}+\beta^{2}+k_{z}^{2}$, the tangential components of the vector $\zeta$ satisfies the following equation $\left(\begin{array}{l}\zeta_{x} \\ \zeta_{y}\end{array}\right)=T\left(\begin{array}{l}E_{0, x} \\ E_{0, y}\end{array}\right)$, and

$$
T=\left(\begin{array}{cccc}
\left(\tau_{\|}-\tau_{\perp}\right) & \cos ^{2} \theta+\tau_{\perp} & \left(\tau_{\|}-\tau_{\perp}\right) & \cos \theta \sin \theta \\
\left(\tau_{\|}-\tau_{\perp}\right) & \cos \theta \sin \theta & \left(\tau_{\|}-\tau_{\perp}\right) & \sin ^{2} \theta+\tau_{\perp}
\end{array}\right) .
$$

$\tau_{\|}, \tau_{\perp}$ represent the Fresnel coefficients of the bare surface, $\theta$ is the angle between $\mathrm{x}$ axis and wave vector $\mathrm{k}$. The third component $\zeta_{z}$ can be deduced from tangential components $\zeta_{z}=-\frac{\alpha \zeta_{i n c, x}+\beta \zeta_{\text {inc,y }}}{\epsilon_{1} k_{0}^{2}-\alpha^{2}-\beta^{2}}$

In our research, the nonlinear effect during laser-metal interaction is negligible since continuous lasers were used. For linear processes, the total energy radiation is proportional to the square of the local electric field magnitude $\boldsymbol{E}\left(\boldsymbol{R}_{\mathbf{0}}, \boldsymbol{r}, \omega\right)$,

$$
\boldsymbol{I}(\boldsymbol{r})=A \chi^{2}(\omega)\left|\boldsymbol{E}\left(\boldsymbol{R}_{\mathbf{0}}, \boldsymbol{r}, \omega\right) \delta \boldsymbol{r}\right|^{2}
$$

In this equation, $\mathrm{A}$ is a constant, $\chi(\omega)$ is the linear susceptibility of the metal, $\delta \boldsymbol{r}$ is the volume element ${ }^{3}$. By applying dynamical Gauss' theorem, the induced electric dipole moment can be written as:

$$
\boldsymbol{P}_{\mathbf{0}}(\boldsymbol{r}, \omega)=\chi(\omega) \boldsymbol{E}_{\mathbf{0}}\left(\boldsymbol{R}_{\mathbf{0}}, \boldsymbol{r}, \omega\right) \delta \boldsymbol{r}
$$

Considering that the metal plasmon was excited by monochromatic circularly polarized light

$$
\boldsymbol{I}(\boldsymbol{r})=P_{0}^{2} \pi^{2} \omega^{2} \rho_{\|}(\boldsymbol{r}, \omega)
$$

$\rho_{\|}(\boldsymbol{r}, \omega)$ represents the in-plane local density of state (LDOS) of plasmonic structure

$$
\rho_{\|}(\boldsymbol{r}, \omega)=\frac{1}{2 \pi^{2} \omega} \operatorname{Im}\left\{S_{x, x}(\boldsymbol{r}, \boldsymbol{r}, \omega)+S_{y, y}(\boldsymbol{r}, \boldsymbol{r}, \omega)\right\} .
$$


$S_{x, x}(\boldsymbol{r}, \boldsymbol{r}, \omega)$ and $S_{y, y}(\boldsymbol{r}, \boldsymbol{r}, \omega)$ are the first two diagonal components of the Green dyadic tensor $S(\boldsymbol{r}, \boldsymbol{r}, \omega)$ at a point $\boldsymbol{r}$ inside the metallic particle. We can deduce the electronic field distribution by identifying the equation (12) and equation (10)

$$
\left|\boldsymbol{E}\left(\boldsymbol{R}_{\mathbf{0}}, \boldsymbol{r}, \omega\right)\right|^{2}=A^{-1} \pi^{2} \omega^{2}\left|\boldsymbol{E}_{\mathbf{0}}\left(\boldsymbol{R}_{\mathbf{0}}, \boldsymbol{r}, \omega\right)\right|^{2} \rho_{\|}(\boldsymbol{r}, \omega)
$$

The electron density can be obtained by Gauss's law

$$
\nabla \cdot \boldsymbol{E}\left(\boldsymbol{R}_{\mathbf{0}}, \boldsymbol{r}, \omega\right)=\frac{\boldsymbol{\rho}}{\varepsilon_{m}} .
$$

For our system the transmitted light, according to Maxwell's equations, the relation between local electronic field and charge density distribution is

$$
\nabla \times \nabla \times \boldsymbol{E}\left(\boldsymbol{R}_{\mathbf{0}}, \boldsymbol{r}, \omega\right)+\frac{1 \partial^{2} \boldsymbol{E}\left(\boldsymbol{R}_{\mathbf{0}}, \boldsymbol{r}, \omega\right)}{c^{2} \partial t^{2}}=-\mu_{0} \frac{\partial \rho v}{\partial t} .
$$

where $v$ is the rate of electron motion.

\section{The relation between electronic field and scattering light signal}

The time-averaged absorption of the material $P_{a b s}\left(\boldsymbol{R}_{\mathbf{0}}, \omega\right)$ can be calculated by the divergence of poynting vector $\nabla \cdot \boldsymbol{S}\left(\boldsymbol{R}_{\mathbf{0}}, \omega\right)^{4}$,

$$
P_{a b s}\left(\boldsymbol{R}_{\mathbf{0}}, \omega\right)=\frac{1}{2} \iint_{V} \omega \varepsilon_{m}^{\prime \prime}\left|\boldsymbol{E}\left(\boldsymbol{R}_{\mathbf{0}}, \boldsymbol{r}, \omega\right)\right|^{2}+\omega \mu^{\prime \prime}\left|\boldsymbol{H}\left(\boldsymbol{R}_{\mathbf{0}}, \boldsymbol{r}, \omega\right)\right|^{2} d V .
$$

For metal, the imaginary part of the magnetic permeability $\mu^{\prime \prime}=0$, so

$$
P_{a b s}\left(\boldsymbol{R}_{\mathbf{0}}, \omega\right)=\frac{1}{2} \iint_{V} \omega \varepsilon_{m}^{\prime \prime}\left|\boldsymbol{E}\left(\boldsymbol{R}_{\mathbf{0}}, \boldsymbol{r}, \omega\right)\right|^{2} d V .
$$

According to energy conservation, for our system the transmitted light $P_{\text {tra }}=0$, the reflection of the gold triangle at the incident light $P_{\text {in }}$ can be written as:

$$
P_{s c a}\left(\boldsymbol{R}_{\mathbf{0}}, \omega\right)=P_{i n}-P_{a b s}-P_{t r a}=-\frac{1}{2} \iint_{V} \omega \varepsilon_{m}^{\prime \prime}\left|\boldsymbol{E}\left(\boldsymbol{R}_{\mathbf{0}}, \boldsymbol{r}, \omega\right)\right|^{2} d V+P_{i n}
$$

Substitute equation (14) into equation (19), then we have

$$
P_{s c a}\left(\boldsymbol{R}_{\mathbf{0}}, \omega\right)=-\frac{1}{2} \iint_{V} A^{-1} \pi^{2} \varepsilon_{m}^{\prime}{ }^{\prime} \omega^{3}\left|\boldsymbol{E}_{\mathbf{0}}\left(\boldsymbol{R}_{\mathbf{0}}, \boldsymbol{r}, \omega\right)\right|^{2} \rho_{\|}(\boldsymbol{r}, \omega) d V+P_{i n}
$$

When the excitation laser spot was approximated as an infinitely small spot and the 
incident light was substracted as background signal, the scattering signal for a given system can be be expressed as:

$$
P_{s c a}\left(\boldsymbol{R}_{\mathbf{0}}, \omega\right) \propto-\omega^{3} \rho_{\|}\left(\boldsymbol{R}_{\mathbf{0}}, \omega\right) .
$$

The scattering light signal obtained by the optical power loss microscopy, which constitutes the plasmonic Chladni pattern, is directly proportional to the in-plane photonic LDOS.

\section{Image processing of the optical power loss microscopy (OPLM) image}

At the most basic principle, the microscope scanning spot can be treated as a point spread function (PSF, h), and the unblurred image also can be treated as a function, $\mathrm{f}$. The process of imaging can be described by the mathematical processing of convolution, $\mathrm{g}=\mathrm{h} \otimes \mathrm{f}$, where $\mathrm{g}$ is the blurred image by the confocal PSF. Using the $80 \mathrm{~nm}$ gold nanoparticle, we captured the confocal PSF which represent the microscope function (Figure S1) and import it into the deconvolution algorithm ${ }^{5,6}$ to obtain the deblurred image.

\section{Comparison of the different methods of investigating the plasmon}

Based on different physical mechanisms, three categories of approaches have been performed to map the modes of plasmon oscillation: (i) the electron beam based methods ${ }^{7}$, such as electron energy loss spectrum (EELS) and cathodoluminescence (CL), (ii) organic molecule-assistant approaches ${ }^{1,8}$, such as enhanced fluorescence, Raman microscopy. (iii) the near- and far- field optical methods, such as two photon luminescence $(\mathrm{TPL})^{9}$, scanning near-field optical microscopy $(\mathrm{SNOM})^{10}$, leakage radiation microscopy $(\mathrm{LRM})^{11}$. 
EELS employs the electron beam rather than light by taking advantage of its high resolution. Based on detecting the inelastic collision between fast electrons and lowenergy valence electrons, electron beam may only excite part of photonic LDOS since it does not have polarization. EELS signal may relate to the Z-component of LDOS as the equation shows

$$
\frac{\Gamma(\boldsymbol{R}, \omega)}{L}=\frac{2 \pi e^{2}}{\hbar \omega} \rho_{z}(\boldsymbol{r}, \omega)
$$

$\Gamma(\boldsymbol{R}, \omega)$ is the electron energy loss probability on the position $\boldsymbol{R}, \boldsymbol{L}$ is the quantization length along $z, \boldsymbol{\rho}_{z}(\boldsymbol{r}, \omega)$ is the Z-component of LDOS at point $\boldsymbol{r}$ in light frequency $\omega^{12}$. Meanwhile, electron beam couples to the surface plasmons through a different way with light, thus a clear-cut relation between EELS and photonic LDOS may not even exists ${ }^{13}$. In addition, electron beam excites dark modes or high order harmonics that cannot be excited optically. CL signal is a part of EELS which decays radiatively. Organic molecule assistant approaches indirectly detect the electronic field hot spots in specific areas, usually on the tip or gap region of nanoparticles. The detected signal of SNOM is strongly correlated with the vertical component of the LDOS.

$$
L D O S=L D O S_{v a c}+\frac{1}{2 \pi^{2} \omega|p|^{2}} \operatorname{Im}\left\{E^{r e f}(\boldsymbol{r}) \cdot \mathrm{p}^{*}\right\}
$$

Where $L D O S_{v a c}$ is the projected LDOS in vacuum and $E^{r e f}(\boldsymbol{r})$ is the field reflected by nearby structures. TPL microscopy provides a far-field excitation and detection without disturbance on the near-field eigenmodes, but it picks up weak signals related to the squared in-plane LDOS, which buries the linear parts that is predominant in lightmetal coupling. The TPL signal can be expressed as 


$$
I_{T P L}(\mathbf{R}, \omega)=\eta^{2}(\omega) \pi^{4} \omega^{4} k \iint_{V}\left|\boldsymbol{E}\left(\boldsymbol{R}_{\mathbf{0}}, \boldsymbol{r}, \omega\right)\right|^{4} \rho_{\|}^{2}(\boldsymbol{r}, \omega) d V
$$

where $\eta(\omega)$ is $\omega$ dependent nonlinear coefficient associated with the metal. As the polarization equation shows, TPL is a three order nonlinear effect linked to the thirdorder nonlinear susceptibility $\chi^{(3)}$

$$
\mathbf{P}=\varepsilon_{0}\left(\chi^{(1)} \cdot \boldsymbol{E}+\chi^{(2)}: \boldsymbol{E} \boldsymbol{E}+\chi^{(3)}: \boldsymbol{E} \boldsymbol{E} \boldsymbol{E}+\cdots\right)
$$

where $\mathbf{P}$ and $\boldsymbol{E}$ are the dielectric polarization density and the electrical field, respectively. $\varepsilon_{0}$ and $\chi^{(n)}$ represent the permittivity in vacuum and $n$-th-order susceptibilities of the medium. The physical foundation of OPLM is a linear effect of light-matter interaction which is linked to $\chi^{(1)}$, therefore, the signal of OPLM is very strong. LRM studies the behavior of surface plasmon polariton by the leakage radiation, therefore, it is not suitable for obtaining details inside the resonator. 


\section{Supplementary figures}

a

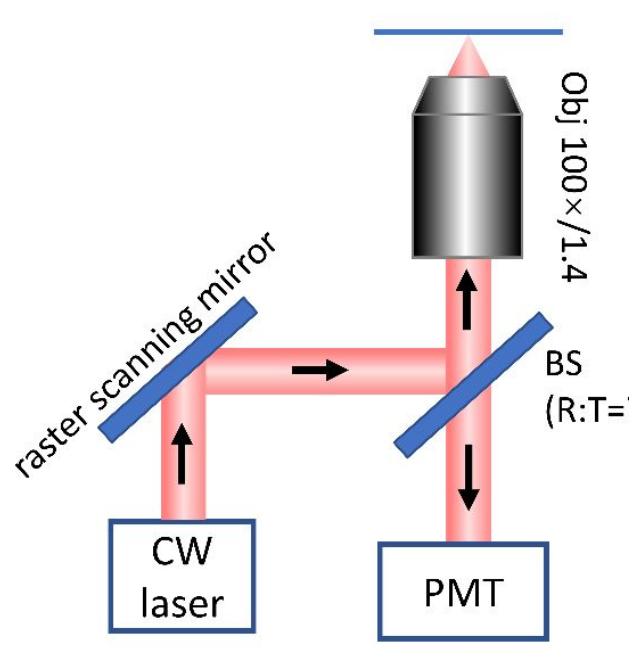

b
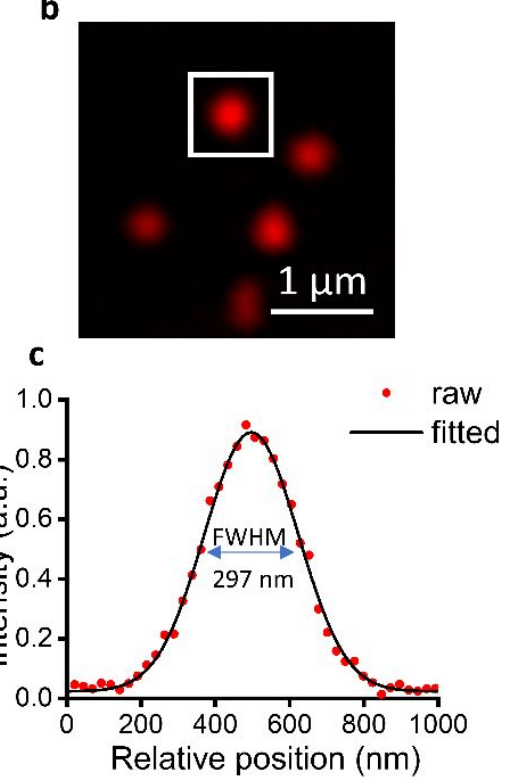

Figure S1. Schematic of the far field optical power loss microscope. a, A continuous wave $(\mathrm{CW})$ laser is reflected by a beam splitter (BS) with a reflection transmission ratio of 7:3. Photomultiplier tube (PMT) captures signals of the same wavelength. $\mathbf{b}$, The point spread function measured by the $80 \mathrm{~nm}$ gold nanoparticles in diameter on OPLM at $633 \mathrm{~nm}$ imaging wavelength. c, Intensity profile extracted from the light spot in (a) in the white box. The fitted profile indicates the resolution of OPLM is $297 \mathrm{~nm}$. 


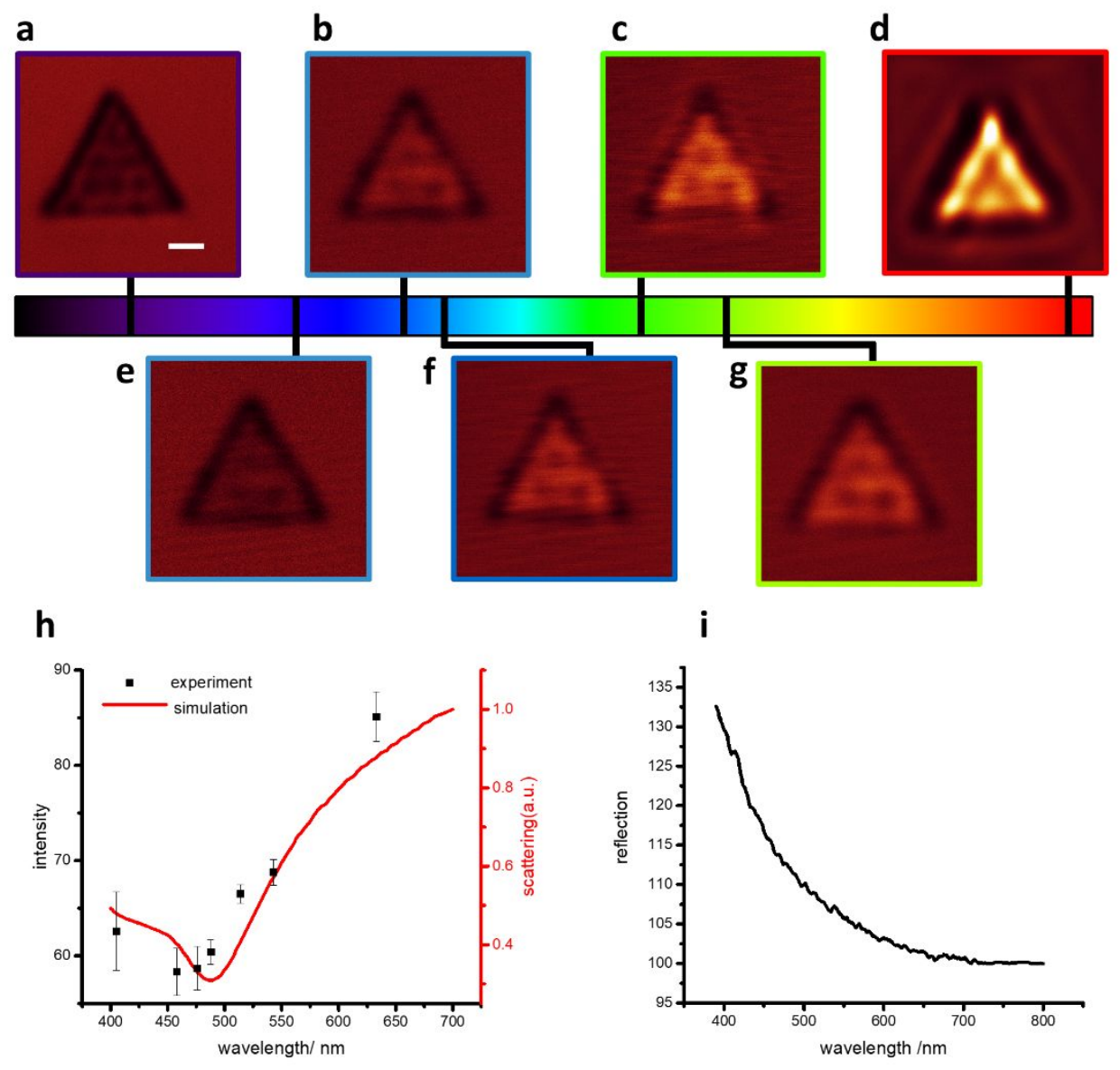

Figure S2. Scattering spectrum of the micro plasmonic Chladni plate. a-g,

Calibrated experimental plasmonic Chladni figure at $\lambda_{\mathrm{exc}}=405 \mathrm{~nm}(\mathbf{a}), \lambda_{\mathrm{exc}}=458 \mathrm{~nm}$ (e), $\lambda_{\text {exc }}=476 \mathrm{~nm}(\mathbf{b}), \lambda_{\text {exc }}=488 \mathrm{~nm}(\mathbf{f}), \lambda_{\text {exc }}=514 \mathrm{~nm}(\mathbf{c}), \lambda_{\text {exc }}=543 \mathrm{~nm}(\mathbf{g}), \lambda_{\text {exc }}$ $=633 \mathrm{~nm}(\mathbf{d})$ on the surface of gold triangle with the edge length of $2 \mu \mathrm{m}(\mathrm{L}=2 \mu \mathrm{m})$. Scale bar, $500 \mathrm{~nm} . \mathbf{h}$, Scattering spectrum of the gold triangle. The square dots and the solid line represent the experimental and normalized simulation results, respectively. i, Silicon substrate reflection spectrum as the baseline to calibrate the plasmonic Chladni figure.

As our experimental system contains a silicon substrate that responses independently 
to incident light, the silicon reflection index provides a convenient reference used to calibrate scattering of gold triangles. Under this calibration, a series of images were obtained under different excitation wavelengths (Figure S2), and scattering spectrum can be obtained by sum the calibrated pixel value of individual image. As the experimental data and the calculated curve showed, the scattered light intensity decreased in the blue end (400 nm-475 nm) of visible spectrum, nevertheless, increased in the red end. The closely matching between experimental and theory data confirmed that the experimental information origin from the scattered light of gold triangle surface. This result is slightly different with the reflection index of the bulk metal due to the coupling effect of the gold triangle edge.
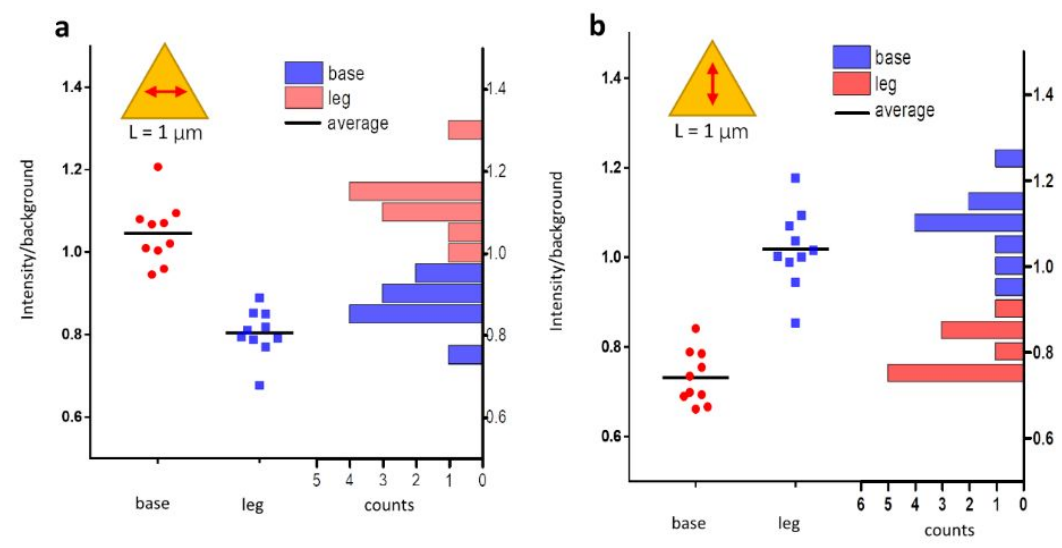

Figure S3. Scattering intensity statistics of gold triangle with the edge length of 1 $\mu \mathrm{m}$ at the $633 \mathrm{~nm}$ excitation wavelength. a, Statistics data from gold triangle edge at polarization direction parallel to the base edge. b, Statistics data from gold triangle edge at polarization direction perpendicular to the base edge. The intensity data normalized by the background intensity of silicon substrate. The red double arrow and the yellow 
triangle represent the direction of polarization and the gold triangle.
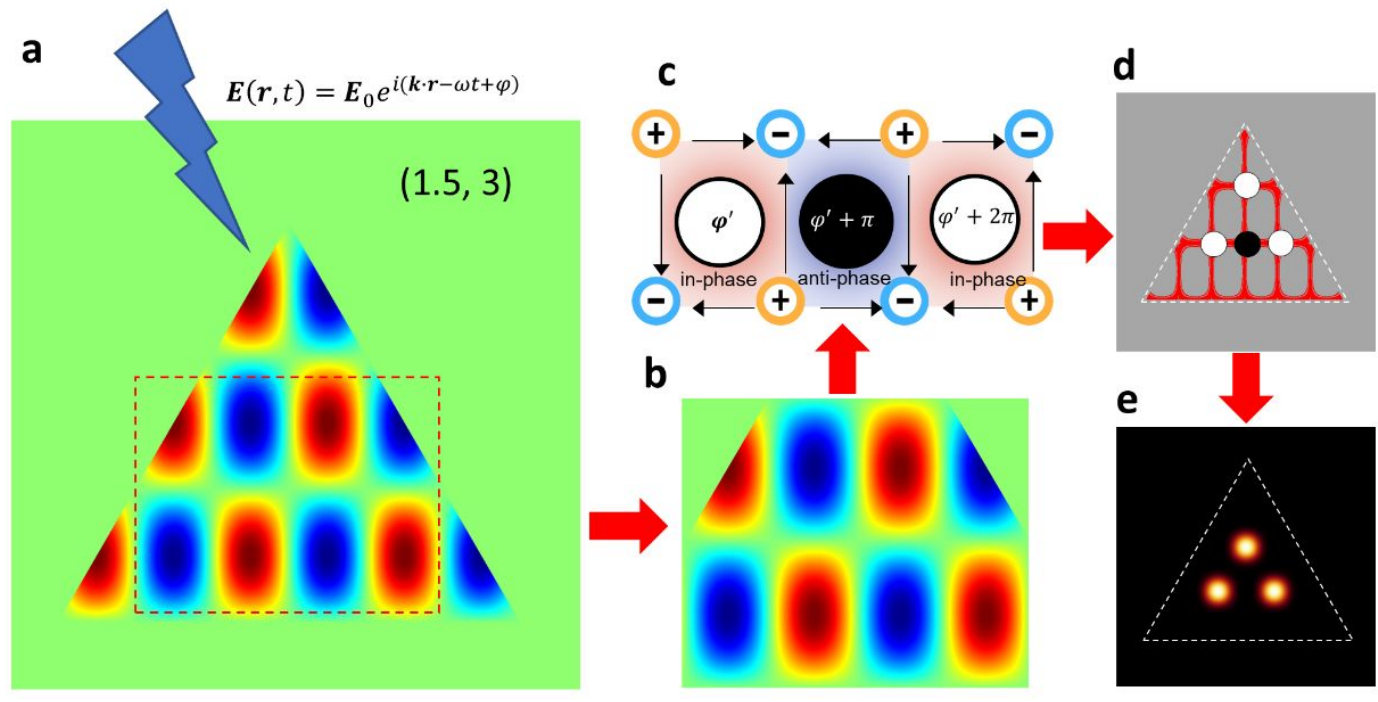

Figure S4. Node selection model based on electric quadrupole. a, The ideal 2D standing $\mathrm{p}-\mathrm{CDW}$ at $\lambda_{\mathrm{exc}}=405 \mathrm{~nm}$ on the surface of gold triangle with the edge length of $1 \mu \mathrm{m}$. b. Interception of three linked electric quadrupoles from a. c, Simplified sketch indicating the in-phase and anti-phase of electric quadrupoles to incident light. d, Extracted nodal pattern from a with marked bright and dark nodes. e, Predicted plasmonic Chladni figure captured by power loss microscope.

\section{Supplementary movies}

Movie 1: Three dimensional muti-angle landscape of experiment plasmon Chladni figure.avi

Movie 2: Two dimensional standing wave.avi

\section{Supplementary references}

1. Novotny, L. \& Hecht, B. Principles of nano-optics, (Cambridge University Press, Cambridge, 2012). 
2. Teulle, A. et al. Scanning optical microscopy modeling in nanoplasmonics. J. Opt. Soc. Am. B. 29, 2431-2437 (2012).

3. Viarbitskaya, S. et al. Tailoring and imaging the plasmonic local density of states in crystalline nanoprisms. Nat. Mater. 12, 426-432 (2013).

4. Ferry, V. E., Sweatlock, L. A., Pacifici, D. \& Atwater, H. A. Plasmonic Nanostructure Design for Efficient Light Coupling into Solar Cells. Nano Lett. 8, 4391-4397 (2008).

5. Biggs, D.S.C. \& Andrews, M. Acceleration of iterative image restoration algorithms. Appl. Opt. 36, 1766-1775 (1997).

6. Hanisch, R.J., White, R.L. \& Gilliland, R.L. Deconvolution of Hubbles Space Telescope images and spectra. in Deconvolution of images and spectra (2nd ed.)(ed. Peter, A.J.) 310-360 (Academic Press, Inc., 1996).

7. García de Abajo, F. J. Optical excitations in electron microscopy. Rev. Mod. Phys. 82, 209-275 (2010).

8. Willets, K. A., Wilson, A. J., Sundaresan, V. \& Joshi, P. B. Super-Resolution Imaging and Plasmonics. Chem Rev. 117, 7538-7582 (2017).

9. Viarbitskaya, S. et al. Tailoring and imaging the plasmonic local density of states in crystalline nanoprisms. Nat. Mater. 12, 426 (2013).

10. Chen, J. et al. Optical nano-imaging of gate-tunable graphene plasmons. Nature 487, 77-81 (2012).

11. Chen, H.-Z. et al. Imaging the dark emission of spasers. Sci. Adv. 3, e1601962 (2017).

12. García de Abajo, F. J. \& Kociak, M. Probing the Photonic Local Density of States with Electron Energy Loss Spectroscopy. Phys. Rev. Lett. 100, 106804 (2008). 
13. Hohenester, U., Ditlbacher, H. \& Krenn, J. R. Electron-Energy-Loss Spectra of Plasmonic Nanoparticles. Phys. Rev. Lett. 103, 106801 (2009). 